\title{
Medidas De Prevención De Infecciones Y Colonizaciones Nosocomiales Por Enterococcus Faecium Resistente A Vancomicina
}

\author{
Sandra Alvarez Hidalgo(MQCD) \\ Universidad Autónoma de Querétaro, Querétaro, México \\ Facultad de Medicina \\ Maestría en Química Clínica Diagnóstica, Facultad de Química \\ Ruth Magdalena Gallegos Torres(PhD) \\ Universidad Autónoma de Querétaro, Querétaro, México \\ Facultad de Enfermería \\ Juan Campos Guillén(PhD) \\ Universidad Autónoma de Querétaro, Querétaro, México \\ Maestría en Química Clínica Diagnóstica, Facultad de Química \\ Maribel Liñan Fernandez(PhD) \\ Aide Teran Alcocer(PhD) \\ Universidad Autónoma de Querétaro, Querétaro, México \\ Facultad de Medicina \\ María Carlota García-G(PhD) \\ Universidad Autónoma de Querétaro, Querétaro, México \\ Facultad de Medicina \\ Maestría en Química Clínica Diagnóstica, Facultad de Química
}

Doi:10.19044/esj.2021.v17n25p193

Submitted: 09 June 2021

Accepted: 24 June 2021

Published: 31 July 2021
Copyright 2021 Author(s)

Under Creative Commons BY-NC-ND 4.0 OPEN ACCESS

Cite As:

Hidalgo S.A., Gallegos Torres R.M., Guillén J.C., Fernandez M.L., Alcocer A.T. \& GarcíaG. M.G.(2021). Medidas De Prevención De Infecciones Y Colonizaciones Nosocomiales Por Enterococcus Faecium Resistente A Vancomicina. European Scientific Journal, ESJ, 17(25), 193.https://doi.org/10.19044/esj.2021.v17n25p193

\section{Resumen}

Las Infecciones Asociadas a la Atención de la Salud (IAAS) son un problema de salud pública global, causado por microorganismos oportunistas, dentro de los cuales los Enterococcus faecium resistentes a vancomicina (ERV) son una de las tres principales especies de bacterias responsables. La 
colonización intestinal por ERV es considerada requisito y factor de riesgo para el desarrollo de estas infecciones. La presencia de ERV se ha asociado a multi-terapia antimicrobiana, largas estancias hospitalarias, inmunosupresión y enfermedades graves. Se ha propuesto que la diseminación intrahospitalaria entre pacientes, por el personal de salud o vía fómites, es el mecanismo de transmisión en infecciones y brotes hospitalarios. Éste es un artículo de revisión que resume las publicaciones en habla inglesa de la última década, acerca de las principales recomendaciones de prevención y control de brotes por ERV. Las medidas para la prevención y contención de la colonización e infecciones por ERV se clasificaron en: 1) medidas de vigilancia epidemiológica y detección temprana; 2) prevención de la colonización y eliminación de reservorios; 3) Manejo de pacientes colonizados y/o infectados; y 4) recomendaciones para el uso razonados de antimicrobianos y tratamiento empírico inicial de las infecciones por ERV. El impacto positivo de la implementación de este tipo de medidas ha sido demostrado por distintos autores.

Palabras Clave: Enterococcus Faecium, Resistencia A Vancomicina, Monitoreo Epidemiolóico, Guías

\title{
Measures Of Prevention And Control Of Vancomycin Resistant Enterococcus Faecium Colonization And Infection
}

\author{
Sandra Alvarez Hidalgo(MQCD) \\ Universidad Autónoma de Querétaro, Querétaro, México \\ Facultad de Medicina \\ Maestría en Química Clínica Diagnóstica, Facultad de Química \\ Ruth Magdalena Gallegos Torres(PhD) \\ Universidad Autónoma de Querétaro, Querétaro, México \\ Facultad de Enfermería \\ Juan Campos Guillén(PhD) \\ Universidad Autónoma de Querétaro, Querétaro, México \\ Maestría en Química Clínica Diagnóstica, Facultad de Química \\ Maribel Liñan Fernandez(PhD) \\ Aide Teran Alcocer(PhD) \\ Universidad Autónoma de Querétaro, Querétaro, México \\ Facultad de Medicina \\ María Carlota García-G(PhD) \\ Universidad Autónoma de Querétaro, Querétaro, México \\ Facultad de Medicina \\ Maestría en Química Clínica Diagnóstica, Facultad de Química
}




\begin{abstract}
Health Care Associated Infections (HAI) are a global public health problem caused by opportunistic microorganisms, among which vancomycin resistant Enterococcus faecium (VRE) are considered one of the three main responsible bacteria. The colonic colonization by VRE is considered a requirement as well as risk factor for the development of these infections. The presence of VRE has been associated with multiple antimicrobial therapy, prolonged hospitalization, immunosuppression, or serious diseases. Intrahospital dissemination between patients, through healthcare workers or via fomites, has been described as the transmission mechanism in endemic infections and hospital outbreaks. This is a review article in Spanish that compiles the English-language publications of the last decade, about the main recommendations for the prevention and control of VRE outbreaks. Recommendations for prevention and control of VRE colonization and infection are classified in: 1 ) epidemiological surveillance and early detection measures; 2) prevention of colonization and reservoirs elimination; 3) management of colonized and / or infected patients; and 4) recommendations for rational use of antimicrobials and initial empirical VRE infection treatment. The positive impact of the implementation of this type of measures has been demonstrated by different authors.
\end{abstract}

Keywords: Enterococcus Faecium,Vancomycin Resistance, Epidemiological Monitoring, Guidelines

\title{
Introducción
}

Enterococcus faecium es una bacteria que normalmente forma parte de la microbiota del sistema digestivo de personas sanas, así como de algunos animales, principalmente mamíferos, siendo un colonizador natural, presentándose en los humanos solo a una concentración menor al 1\% (Contreras, Munita, \& Arias, 2019). Sin embargo, bajo ciertas circunstancias, adquisición de resistencia antimicrobianos, puede convertirse en un patógeno oportunista capaz de generar infecciones en personas hospitalizadas, traspasando las barreras intestinales e infectando otros órganos, provocando bacteriemia, infección en vías urinarias, endocarditis, infecciones intraabdominales y pélvicas, infecciones de la piel y en algunas ocasiones infecciones del sistema nervioso central (Arias \& Murray, 2012; Contreras et al., 2019; Mutters et al., 2013).

Los Enterococcus resistentes a vancomicina (ERV) ocupa entre el segundo y tercer lugar dentro de los patógenos causantes de Infecciones Asociadas a la Atención de la Salud, por lo que es considerado un problema de salud mundial, afectando principalmente a personas con 
inmunocompromiso, largas estancias hospitalarias y uso prolongado de terapia antimicrobiana (O’Driscoll \& Crank, 2015).

La emergencia de ERV como patógeno se ha atribuido principalmente a su capacidad de colonizar el tracto gastrointestinal de pacientes que han sido hospitalizados por largos periodos de tiempo y que han estado bajo terapia antimicrobiana, debido a que el uso de antibióticos inhibe la microbiota benigna, permitiendo así la invasión intestinal por ERV, volviéndose una situación decisiva para su transmisión y la adquisición de resistencia antimicrobiana (Contreras, 2019).

Adicionalmente, esta bacteria tiene una gran capacidad de sobrevivir y permanecer por periodos prolongados en el ambiente hospitalario, aunado a su plasticidad genómica y presencia de elementos genéticos móviles que le confieren capacidades adaptativas y de resistencia que lo convierten en un patógeno de difícil manejo (Isenman \& Fisher, 2016; O’Driscoll \& Crank, 2015; Orsi \& Ciorba, 2013; Satilmis, Vanhems, \& Bénet, 2015).

Entre los factores de riesgo descritos para que un paciente curse colonización y posible posterior infección por EVR se incluyen: edad, inmunocompromiso, uso prolongado de antimicrobianos, larga estancia hospitalaria en Unidades de Cuidados Intensivos, presencia de dispositivos médicos invasivos (catéteres, sondas), cirugías y la movilización de los pacientes colonizados o infectados entre distintas áreas del hospital (Chiang et al., 2017; Djembi et al., 2017; Gilbert et al., 2017; Mahony et al., 2018).

La propagación de EVR se ha asociado a su presencia principalmente en instrumental médico y mobiliario hospitalario, los cuales actúan como fómites. A su vez, las infecciones debidas a este microorganismo se asocian a largas estancias hospitalarias, alta mortalidad, readmisión y altos costos hospitalarios (Chiang et al., 2017; Miller, Munita, \& Arias, 2014; Miller, Murray, Rice, \& Arias, 2016).

Además de la gran capacidad de Enterococcus faecium de adquirir multiresistencia a antibióticos, también tiene la capacidad de adaptarse fácilmente y resistir a condiciones ambientales adversas de temperatura, $\mathrm{pH}$ y concentración de sales, etc. El incremento en las infecciones nosocomiales por enterococos resistente a glucopéptidos como la vancomicina, es considerado un grave problema de salud, debido a la limitación de la terapia de antibióticos. Dichas infecciones se han diseminado alrededor del mundo, aumentando la morbilidad, la mortalidad y los costos de hospitalización (Faron, Ledeboer, \& Buchan, 2016; Isenman \& Fisher, 2016; O’Driscoll \& Crank, 2015; Reyes, Bardossy, \& Zervos, 2016).

El objetivo del presente documento es proponer una compilación, en idioma español, de las principales recomendaciones para la vigilancia, prevención y control de la infección y colonización por Enterococcus spp. resistentes a vancomicina, basado en la literatura internacional, para lo cual se 
realizó una búsqueda en la literatura mundial de la última década en idioma inglés. Se inicia con un panorama histórico y epidemiológico de ERV, culminando con el impacto que ha tenido la implementación de estas medidas en distintos países.

\section{Texto principal}

Historia y epidemiología de Enterococcus faecium resistente a vancomicina

Las cepas de enterococci resistentes a vancomicina fueron identificadas por primera vez en Europa en la década de 1980; su resistencia fue asociada al uso de avoparcina, un glucopéptido utilizado para favorecer el crecimiento del ganado. Posteriormente ERV fue identificado en los Estados Unidos en donde en la década de 1990 incrementó rápidamente su transmisión para finalmente distribuirse por todo el mundo (Levitus, Rewane, \& Perera, 2021). Para la especie E. faecium se conocen dos grupos principales, el clado hospitalario y el clado comunitario; los microorganismos pertenecientes al clado hospitalario tienen una mayor capacidad de diseminarse en los ambientes nosocomiales (Faron et al., 2016; Miller et al., 2014, 2016; O’Driscoll \& Crank, 2015; Reyes et al., 2016).

Los enterococos son la tercera causa más común de infección como la endocarditis y fue a finales de 1940 cuando se propuso el uso combinado de penicilina y un aminoglucósido como parte del tratamiento de ésta. Actualmente, la resistencia a los aminoglucósidos se ha incrementado, por lo cual dicho tratamiento ya no es eficiente contra algunas cepas (Faron et al., 2016; Miller et al., 2016; Reyes et al., 2016).

La ocurrencia de infecciones por ERV ha ido en aumento; por ejemplo en Estados Unidos, el incremento en el aislamiento de esta bacteria ha sido de más del 90\% (Chiang et al., 2017). Se considera que esta bacteria ha adquirido características genéticas que contribuyen al éxito en su prevalencia en ambientes hospitalarios, adicional al desarrollo y adquisición de determinantes de resistencia a múltiples antimicrobianos (Faron et al., 2016; Miller et al., 2014, 2016; O’Driscoll \& Crank, 2015; Reyes et al., 2016; Whang, Miller, Partain, \& McKinnell, 2013).

Actualmente se sabe que genotipo vanA tiene un origen plasmídico pero es codificado por un transposon vanA y por lo tanto puede incorporarse al cromosoma; por el contrario, el gen $v a n B$ es codificado por un transposon $v a n B$ conjugativo y también puede ser trasferido a otras cepas por medio de un plásmido. El genotipo vanA confiere resistencia a vancomicina y teicoplanina mientras que el genotipo vanB solo a la vancomicina (Faron et al., 2016; Miller et al., 2014, 2016; Reyes et al., 2016).

La mayoría de los casos de E. faecium resistente a vancomicina en los Estados Unidos, Europa, Corea, América del Sur y África se deben 
actualmente al genotipo vanA. En cambio, en el caso de Australia y Singapur son ocasionados principalmente por el genotipo vanB. La tasa de diseminación de E. faecium V varía mucho, pero el mayor porcentaje de ERV se ha registrado en los EE.UU., Irlanda y Australia (Miller et al., 2016; Reyes et al., 2016; Talaga-Ćwiertnia \& Bulanda, 2018).

En Australia se ha rastreado una nueva secuencia tipo de E. faecium ERV, la ST796, la cual se considera la causa principal de bacteriemia. La secuenciación completa del genoma (WGS) ha demostrado una estructura poblacional esencialmente clonal de ST796, pero a diferencia de las bacterias gram negativas resistentes a múltiples medicamentos, al parecer, su éxito no se debe a una mayor resistencia a los antibióticos. Esto sugiere que otros determinantes han impulsado su rápida diseminación como la posibilidad de resistencia a los biocidas (Mahony et al., 2018).

Las infecciones causadas por ERV constituyen una amenaza relevante para la salud pública, por lo tanto es necesario contar con guías de vigilancia y programas destinados a la detección temprana, con el fin de prevenir su propagación en la población hospitalaria. La adherencia a este tipo de programas ha demostrado tener un impacto en la prevención del desarrollo de brotes hospitalarios (Cheng et al., 2016; Chiang et al., 2017; Djembi et al., 2017; Lai CK, Wong SY, Lee SS, Siu HK, Chiu CY, Tsang DN, Ip MP, 2017; Lemieux et al., 2017; Talaga-Ćwiertnia \& Bulanda, 2018; Ulrich \& Gastmeier, 2017).

Los Departamentos de Control de las Infecciones Nosocomiales son los grupos conformados por enfermeras, epidemiólogos y/o infectólogos, clínicos y administradores de servicios en salud que coordinan y supervisan las actividades de detección, investigación, registro, notificación y análisis de información, además de la capacitación para la detección, manejo y control de las infecciones nosocomiales (cdc, 2010; Diario Oficial de la Federación, 2009; Flanagan, Cassone, Montoya, \& Mody, 2016; Frakking et al., 2018; Montoya, Cassone, \& Mody, 2016; Ulrich \& Gastmeier, 2017).

A continuación se presentan las recomendaciones de vigilancia activa para la prevención y control de la infección y colonización por ERV. Dichas medidas deben realizarse de manera rutinaria en unidades de cuidados intensivos, pacientes trasplantados, con diálisis crónica, y en unidades con largas estancias hospitalarias. Adicionalmente deben establecerse estas medidas ante un brote de infección en cualquier área hospitalaria (Cheng et al., 2016; Lai CK, Wong SY, Lee SS, Siu HK, Chiu CY, Tsang DN, Ip MP, 2017; Reyes et al., 2016). 


\section{Recomendaciones para la vigilancia epidemiológica y detección temprana de colonización intestinal por ERV}

- La detección de enterococos resistentes a glucopéptidos en heces fecales de los pacientes que cumplan con una estancia hospitalaria mayor de 72 horas (Faron et al., 2016; Flanagan et al., 2016; Lai CK, Wong SY, Lee SS, Siu HK, Chiu CY, Tsang DN, Ip MP, 2017; Montoya et al., 2016; Reyes et al., 2016).

- $\quad$ Para que un paciente pueda considerarse negativo a la colonización por ERV, es necesario obtener un mínimo de tres cultivos negativos realizados semanalmente (Battipaglia et al., 2019), o 5 cultivos negativos de hisopado rectal, tomados en diferentes días posteriores al término de la exposición potencial (Contreras et al., 2019; Frakking et al., 2018).

- $\quad$ Algunos autores consideran importante hacer un muestreo ambiental de superficies y fómites para la identificación de reservorios artificiales, así como de las manos del personal sanitario y de los familiares (Flanagan et al., 2016; Lowden, Miller Neilan, \& Yahdi, 2014; Montoya et al., 2016; Reyes et al., 2016).

- Como parte de la vigilancia epidemiológica para identificar la presencia de ERV así como el tipo de cepa, los métodos de identificación pueden incluir técnicas microbiológicas o biomoleculares (Cheng et al., 2016; Faron et al., 2016; Reyes et al., 2016; Tan, Jiang, \& Ng, 2017):

o Cultivo en medios selectivos (agar entecoccosel suplementado con vancomicina, cromogénicos, entre otros) (Faron et al., 2016; Reyes et al., 2016; Tan et al., 2017).

o Un pre-enriquecimiento de la muestra en caldos de uso común (cerebro-corazón, soya tripticasa, M17) por 4-6 h, disminuye a la mitad el tiempo de identificación de colonias presuntivas en los agares selectivos (Domig, Mayer, \& Kneifel, 2003).

o En los lugares en los que se cuente con la infraestructura y personal capacitado, el uso de métodos biomoleculares permite la identificación de ERV colonizantes en menos de 24 h (Faron et al., 2016; Ozback, 2018; Reyes et al., 2016; Tan et al., 2017).

- Realizar la tipificación molecular de los aislamientos de enterococos resistentes a glucopéptidos para clarificar la epidemiología de este microorganismo (Frakking et al., 2018; Grabsch et al., 2012; Mahony et al., 2018; Reyes et al., 2016).

- Para el análisis de la relación filogenética entre las cepas, se recomienda utilizar la secuenciación completa del genoma (WGS), la cual ofrece una diferenciación más precisa de cada cepa que la técnica de PFGE (Electroforesis en Gel con Campos Pulsados), y se empieza a utilizar de una manera más constante en las investigaciones de brotes por ERV (Frakking et al., 2018; Grabsch et al., 2012; Mahony et al., 2018; Reyes et al., 2016). 
- También se recomienda el uso de la técnica de Tipificación Multilocus de Secuencias (MLST) para identificar la presencia de cepas que pertenezcan al complejo clonal 17 (CC17), complejo que agrupa linajes de Enterococcus faecium aislados de pacientes hospitalizados (Contreras et al., 2019; Freitas et al., 2010; Lee, Pang, Abraham, \& Coombs, 2019).

\section{Recomendaciones de prevención de colonización y eliminación de reservorios (educación, higiene, instrumentos de barrera, limpieza)}

- $\quad$ La higiene de manos es fundamental antes y después del contacto con cada paciente. El jabón por sí solo puede no ser suficiente, es mejor utilizar soluciones de jabón y desinfectante. El alcohol líquido o en gel es más conveniente que el uso de otros desinfectantes; éstas medidas aplican para el personal médico y para los familiares del paciente (Barnes, Morgan, Harris, Carling, \& Thom, 2014; Bryce et al., 2015; Cheng et al., 2016; Fernando, Gray, \& Gottlieb, 2017; Kecik Bosnak, Namiduru, Karaoglan, \& Ozlem Mete, 2013; Lai CK, Wong SY, Lee SS, Siu HK, Chiu CY, Tsang DN, Ip MP, 2017). - Las manos del personal médico deben ser descontaminadas en los 5 momentos para la higiene de manos y según los criterios de la Organización Mundial de la Salud (OMS) (cdc, n.d.; Rutala, Weber, Healthcare, \& Infection Control Practices Advisory Committee, 2017).

- Evitar el uso de uñas artificiales y anillos, pulseras y relojes del personal médico, al tener contacto directo con los pacientes (Francisco, Aragão, \& Cardoso, 2018; Reyes et al., 2016).

- $\quad$ El uso y recambio de batas, de preferencia desechables, y guantes es obligatorio al ingresar a las unidades de cuidados intensivos o al manipular secreciones corporales. El recambio y/o limpieza entre pacientes también se recomienda cuando se utilizan equipos de atención relacionada con el paciente, como estetoscopios y manguitos de presión arterial (Fernando et al., 2017; Martin et al., 2016; Morgan DJ, Murthy R, Munoz-Price LS, Barnden M, Camins BC, Johnston BL \& Z, Sullivan KV, Shane AL, Dellinger EP, Rupp ME, 2015; Mutters et al., 2013; Rubin, Samore, \& Harris, 2018). Sin embargo el uso de este tipo de protección no sustituye otras medidas (Fernando et al., 2017; Martin et al., 2016; Morgan DJ, Murthy R, MunozPrice LS, Barnden M, Camins BC, Johnston BL \& Z, Sullivan KV, Shane AL, Dellinger EP, Rupp ME, 2015; Reyes et al., 2016; Rubin et al., 2018).

- Baño diario con toallas impregnadas en clorhexidina a pacientes en unidades de cuidados intensivos. Esta medida no es necesaria de manera rutinaria en otros servicios (Fernando et al., 2017; Kim et al., 2016; Mendes et al., 2016; Reyes et al., 2016).

- $\quad$ Las salas del hospital deben limpiarse regularmente como parte de un programa general de higiene hospitalaria (Bryce et al., 2015; Cheng et al., 
2016; Lai CK, Wong SY, Lee SS, Siu HK, Chiu CY, Tsang DN, Ip MP, 2017; Rutala et al., 2017).

- Después de un brote o incidente de colonización o infección, las habitaciones de aislamiento o la totalidad de una sala después de brotes más extensos, deben ser limpiadas a fondo para reducir la contaminación del medio ambiente (Fernando et al., 2017; Rutala et al., 2017).

- Los equipos contaminados con sangre, fluidos corporales, secreciones o excreciones, deben limpiarse y desinfectarse después de su uso. La misma pauta aplica para todas las superficies, por ejemplo, barandillas, carros, cómodas, perillas de puertas y manijas de lavamanos. Se recomienda el uso de sustancias indicadas para patógenos que pueden sobrevivir en un ambiente inanimado por periodos prolongados (Fernando et al., 2017). Se recomiendan utilizar los siguientes compuestos: fenólicos, sales cuaternarias de amonio, dicloro-isocianurato de sodio e hipoclorito de sodio para superficies, y para equipos médicos los compuestos de dicloro-isocianurato (Fernando et al., 2017; Rutala et al., 2017).

- El proceso de descontaminación se debe realizar después de la limpieza. La solución de hipoclorito a una concentración del 6\% y de alcohol al $70 \%$ son recomendadas para este proceso; llevar bitácoras para la realización de la limpieza y descontaminación rutinaria (Lai CK, Wong SY, Lee SS, Siu HK, Chiu CY, Tsang DN, Ip MP, 2017; Mutters et al., 2013; O’Driscoll \& Crank, 2015; Reyes et al., 2016).

- $\quad$ Se deben descontaminar las franelas con las que se realiza la limpieza, en una solución de hipoclorito durante 2 minutos. Si son franelas reutilizables o trapeadores de limpieza, deben descontaminarse con regularidad para evitar la contaminación durante la limpieza, con la posterior transferencia de organismos entre los pacientes o equipos por las manos de los trabajadores de salud (Rutala et al., 2017).

- Se debe realizar una limpieza y desinfección exhaustiva con regularidad en las áreas de la unidad, para eliminar reservorios ambientales de EVR, asimismo llevar registro en bitácoras de este tipo de limpieza (Bryce et al., 2015; Cheng et al., 2016; Lai CK, Wong SY, Lee SS, Siu HK, Chiu CY, Tsang DN, Ip MP, 2017; Rutala et al., 2017). El beneficio de otros métodos que no requieren contacto, tales como rayos UV, vapor de peróxido de hidrogeno, para la desinfección de superficies e instrumental también ha sido demostrado (Marra, Schweizer, \& Edmond, 2018; Reyes et al., 2016).

- Uno de los factores de riesgo más importante para el desarrollo de infección en pacientes colonizados es el uso de dispositivos médicos de apoyo. Las personas con ventilación mecánica durante más de cinco días incrementaron 10,6 veces el riesgo de contraer infecciones resistentes. En relación con el uso de este dispositivo, se recomienda manipular asépticamente los circuitos, evitar la reintubación, cambiar los circuitos de 
ventilación cuando se ensucien o dañen, cambiar la fijación del tubo endotraqueal y realizar lavados con cloherxedina cada 6 h. Se deberá además, ubicar a los pacientes en una habitación individual, con los equipos básicos requeridos para evitar el contagio directo o indirecto (Fernando et al., 2017; Kim et al., 2016; Mendes et al., 2016; Reyes et al., 2016).

- Educación y capacitación constante al personal específico a cargo de unidades de cuidados intensivos, así como de todo el personal que labora en los nosocomios, respecto a la diseminación de bacterias multi-fármaco resistentes, para el control de las infecciones, para lo cual se sugiere la creación de programas de capacitación permanente centrados en la prevención de la contaminación ambiental y la propagación de agentes patógenos (Fernando et al., 2017; Frakking et al., 2018; Montoya et al., 2016).

- $\quad$ Educación en cuanto a las buenas prácticas higiénicas de manos, tanto para el personal sanitario, como de los familiares del paciente (Fernando et al., 2017; Frakking et al., 2018; Montoya et al., 2016).

- Conferencias didácticas, además de información para ser difundida en el hospital respecto a las medidas adecuadas de control de infecciones y el uso de glucopéptidos (Fernando et al., 2017; Flanagan et al., 2016; Frakking et al., 2018).

\section{Recomendaciones para el manejo de colonización y/o infección (aislamiento y traslado)}

- Con base en los resultados de la vigilancia activa, se realizará la clasificación de los pacientes en: no colonizados, colonizados e infectados, para el manejo en aislamiento de los dos últimos (Frakking et al., 2018; Lai CK, Wong SY, Lee SS, Siu HK, Chiu CY, Tsang DN, Ip MP, 2017).

- Cuando surja un brote de infección por ERV se debe informar al personal para prevenir riesgos de diseminación, así como clasificar como colonización, infección esporádica, infección endémica o epidémica (Lai CK, Wong SY, Lee SS, Siu HK, Chiu CY, Tsang DN, Ip MP, 2017; Satilmis et al., 2015; Ulrich \& Gastmeier, 2017).

- Los pacientes deben aislarse en habitaciones individuales. Más si presentan diarrea o incontinencia, debido a que el riesgo de diseminación es mayor (Frakking et al., 2018; Lai CK, Wong SY, Lee SS, Siu HK, Chiu CY, Tsang DN, Ip MP, 2017).

- $\quad$ Si un paciente con ERV se transfiere a otra institución de salud, se deberá informar a la recepción de la nueva clínica y al personal de control de infecciones (Bryce et al., 2015; Cheng et al., 2016; Lai CK, Wong SY, Lee SS, Siu HK, Chiu CY, Tsang DN, Ip MP, 2017).

- $\quad$ El transplante de microbiota fecal (FMT por sus siglas en inglés), es considerado una de las estrategias de descolonización más prometedora, por medio de la cual se han obtenido resultados favorables al reducir la 
concentración de ERV y en algunos casos, su eliminación completa del intestino de los pacientes tratados (Contreras et al., 2019). Para lograr obtener mejores resultados, se recomienda llevar a cabo el FMT de forma coordinada con la administración de antibióticos y el suministro de probióticos para evitar una reinfección, así como seguir las medidas de limpieza y desinfección ambiental y el aislamiento del paciente (Cheng et al., 2014).

- Otro procedimiento muy innovador para eliminar bacterias multiresistentes, es el uso de bacteriófagos. Se han obtenido resultados favorables cuando el tratamiento se lleva a cabo combinando diversos fagos y administrando al mismo tiempo antibióticos, disminuyendo de ese modo la mortalidad de una manera considerable (Gelman et al., 2018).

- El seguimiento de los pacientes tras el alta es recomendable, para monitorear la persistencia de colonización (Frakking et al., 2018).

- $\quad$ Se deben tomar en cuenta los factores de riesgo para un diagnóstico más personal. Los factores de riesgo que son importantes en la mortalidad de los pacientes son la edad avanzada, la presencia de enfermedades como el cáncer, la existencia de comorbilidades, la gravedad de la infección, los cultivos de sangre positivos e infección por otros patógenos del grupo ESKAPE (Enterococcus faecium, Staphylococcus aureus, Klebsiella pneumoniae, Acinetobacter baumannii, Pseudomonas aeroginosa y Enterobacter spp) (Francisco et al., 2018).

\section{Recomendaciones para el tratamiento empírico temprano de infecciones por ERV y uso razonado de antimicrobianos (uso de antimicrobianos)}

- $\quad$ La aparición y propagación de ERV parece ser intensificada por el uso de agentes antimicrobianos, especialmente glucopéptidos y cefalosporinas (Gilbert et al., 2017). Por lo tanto, todos los hospitales deben tener políticas establecidas para el uso prudente de antibióticos en todas las áreas de la clínica (Fernando et al., 2017; Flanagan et al., 2016; Montoya et al., 2016; Reyes et al., 2016).

- Considerar que la resistencia a glucopéptidos reduce las opciones terapéuticas contra una sepsis por enterococos. La elección de la terapia antimicrobiana debe ser guiada por pruebas previas de sensibilidad a los antibióticos (Fernando et al., 2017; Gilbert et al., 2017; Reyes et al., 2016).

- $\quad$ El uso razonado de antimicrobianos es el principal factor modificable para combatir la resistencia antimicrobiana Los programas de optimización de uso de antimicrobianos (antimicrobial stewardship) incluyen dosis, ruta de administración y duración del tratamiento antimicrobiano, así como la reducción de su uso innecesario. Estos programas deben garantizar la prescripción del antimicrobiano preciso, en el momento y para el diagnóstico correcto (Fernando et al., 2017; Flanagan et al., 2016; Montoya et al., 2016; Reyes et al., 2016). 
- Las estrategias de preautorización y restricción de antibióticos, combinadas con educación, ciclos de auditoría y retroalimentación, son piedra angular de los programas de optimización de uso de antimicrobianos (Fernando et al., 2017; Flanagan et al., 2016; Reyes et al., 2016).

- Reducción del tiempo predeterminado de terapia y revaloración para su seguimiento o cambio de terapia (Fernando et al., 2017; Flanagan et al., 2016; Reyes et al., 2016).

- $\quad$ Preferir la vía de administración oral (Fernando et al., 2017; Flanagan et al., 2016; Reyes et al., 2016).

- $\quad$ Para el tratamiento de bacteriemia por ERV se ha sugerido el uso de daptomicina o linezolid con resultados similares (Isenman \& Fisher, 2016; Zhao et al., 2016). Es importante considerar que algunas cepas con sensibilidad limítrofe a daptomicina presentan mutaciones y se comportan de manera similar a cepas resistentes, por lo que el uso de daptomicina en estas cepas debe evitarse (Munita et al., 2014; Shukla et al., 2016).

- Otros autores consideran que el uso de linezolid no es lo más recomendado contra la bacteriemia por ERV, debido a la actividad bacteriostática y en cambio sugieren el uso de la daptomicina contra infecciones severas pero a concentraciones elevadas de entre $10-12 \mathrm{mg} / \mathrm{kg}$ o utilizar la daptomicina en combinación con $\beta$-lactamos (Foolad, Taylor, Shelburne, Arias, \& Aitken, 2018; Hall, Steed, Arias, Murray, \& Rybak, 2012; Kebriaei et al., 2018).

- $\quad$ El tratamiento con quinupristina-dalfopristina o tigeciclina puede ser usado en casos de falla de tratamiento con daptomicina o linezolid (Isenman \& Fisher, 2016).

- Por último, se recomienda la capacitación en salud al personal asistencial y a los familiares para facilitar la adopción de los protocolos, el cumplimiento de las normas de aislamiento, el seguimiento de los pacientes infectados para disminuir así las estadísticas de infección (Fernando et al., 2017; Londoño Restrepo, Macias Ospina, \& Ochoa Jaramillo, 2016; Mutters et al., 2013; Rutala et al., 2017).

\section{Impacto de las medidas de vigilancia y control de colonización e infección por Enterococcus faecium resistente a vancomicina}

La importancia de tomar medidas precautorias en casos de brotes de infección por ERV ha sido demostrada en diversos estudios. Urlich y Gastmeier (2017), analizando la información de la base de datos de brotes multinacional "www.outbreakdatabase.com" realizaron una investigación comparativa sobre las medidas de control de infecciones por ERV utilizadas en diversos hospitales. Compararon el éxito de dichas medidas enfocadas a un brote altamente endémico y brotes de corta duración, así como si los brotes eran monoclonales o policlonales. En dicho estudio no se pudieron identificar 
diferencias relevantes entre las medidas de control aplicadas de acuerdo al tipo de brote, por lo que consideran que la distinción entre los brotes es importante con fines epidemiológicos, sin embargo no es relevante para la implementación de las medidas de control; es decir, que las medidas podrían generalizarse sin importar el tipo de brote.

Además consideran que no solo el tipo de medidas que se implementen es lo importante, sino la forma y rigurosidad en que fueron implementadas. La única medida de control que resultó significativa entre algunos hospitales, fue el lavado de manos, el cual se puede considerar como una medida fundamental para el control de ERV. También estos autores consideran que es importante detectar de manera temprana a los pacientes con ERV para poder prevenir su propagación entre los pacientes. En promedio, 4.5 medidas de control de infección por brote fueron empleadas (rango 1 a 9). La medida más frecuente adoptada fue la detección de ERV en los pacientes (en 28 brotes), seguido por el aislamiento físico de los pacientes en 21 brotes. En 14 brotes involucrados se hizo la evaluación ambiental y en 15 brotes se intensificó la limpieza y la desinfección del medio ambiente y en seis brotes se implementó el cierre de la ubicación afectada (Urlich y Gastmeier, 2017b).

Diversos estudios han demostrado que dentro de las principales medidas de control y prevención de las infecciones por este microorganismo se encuentra el lavado de manos, y el control de la colonización (Ajao et al., 2011; Grabsch et al., 2012; Mendes et al., 2016; Ziakas, Thapa, Rice, \& Mylonakis, 2013). Asimismo, integrar un Comité de Medicamentos y Terapéutica en trabajo conjunto con el Comité de Vigilancia Epidemiológica, formado por un grupo interdisciplinario con experiencia en el control de las infecciones asociadas a la atención de la salud (IAAS), permitirá dar el seguimiento a las diversas medidas de control y prevención, incluyendo la instauración del uso racional de antibióticos (cdc, n.d.; Diario Oficial de la Federación, 2009; Frakking et al., 2018; Londoño Restrepo et al., 2016; Montoya et al., 2016).

\section{Conclusión}

En conclusión, las recomendaciones de control y prevención de infecciones y colonizaciones nosocomiales por Enterococcus faecium resistente a vancomicina se pueden resumir en: 1) acciones de vigilancia epidemiológica y detección temprana, 2) medidas de prevención de colonización y eliminación de reservorios, 3) manejo de colonización y/o infección, y 4) el tratamiento empírico temprano de infecciones por ERV y uso razonado de antimicrobianos. Es importante que las instituciones sanitarias cuenten con estas medidas de control y prevención de la colonización e infección por ERV, las cuales deben de ser reguladas por un 
comité multidisciplinario ad hoc, quien decidirá y dará seguimiento al conjunto de medidas que considere prioritario en cada caso.

\section{Agradecimiento}

El desarrollo de este trabajo recibió financiamiento por parte de Fondo Sectorial de Investigación en Salud y Seguridad Social SS/IMSS/ISSSTECONACYT, Fondo para el Fortalecimiento de la Investigación-UAQ y Becas Conacyt para Posgrado PNPC. Se agradece el apoyo para la búsqueda bibliográfica a Hernández Vázquez y Ortega Ramírez y a Rodrigo González Sánchez por la revisión crítica.

\section{References:}

1. Ajao, A. O., Harris, A. D., Roghmann, M.-C., Johnson, J. K., Zhan, M., McGregor, J. C., \& Furuno, J. P. (2011). Systematic review of measurement and adjustment for colonization pressure in studies of methicillin-resistant Staphylococcus aureus, vancomycin-resistant enterococci, and clostridium difficile acquisition. Infection Control and Hospital Epidemiology, 32(5), 481-489. https://doi.org/10.1086/659403

2. Arias, C. A., \& Murray, B. E. (2012). The rise of the Enterococcus: beyond vancomycin resistance. Nature Reviews Microbiology, 10(4), 266-278. https://doi.org/10.1038/nrmicro2761

3. Barnes, S. L., Morgan, D. J., Harris, A. D., Carling, P. C., \& Thom, K. A. (2014). Preventing the transmission of multidrug-resistant organisms: modeling the relative importance of hand hygiene and environmental cleaning interventions. Infection Control and Hospital Epidemiology, 35(9), 1156-1162. https://doi.org/10.1086/677632

4. Battipaglia, G., Malard, F., Rubio, M. T., Ruggeri, A., Mamez, A. C., Brissot, E., ... Mohty, M. (2019). Fecal microbiota transplantation before or after allogeneic hematopoietic transplantation in patients with hematologic malignancies carrying multidrug-resistance bacteria. Haematologica, 104(8), 1682-1688. https://doi.org/10.3324/haematol.2018.198549

5. Bryce, E., Grant, J., Scharf, S., Dempster, L., Lau, T. T. Y., Laing, F., ... Forrester, L. (2015). Horizontal infection prevention measures and a risk-managed approach to vancomycin-resistant enterococci: An evaluation. American Journal of Infection Control, 43(11), 12381243. https://doi.org/10.1016/j.ajic.2015.06.003

6. cdc. (2010). Vancomycin-resistant Enterococci (VRE) and the Clinical Laboratory | HAI | CDC. Retrieved October 7, 2020, from https://www.cdc.gov/hai/settings/lab/vreclinical-laboratory.html 
7. Cheng, V. C. C., Chen, J. H. K., Tai, J. W. M., Wong, S. C. Y., Poon, R. W. S., Hung, I. F. N., ... Yuen, K.-Y. (2014). Decolonization of gastrointestinal carriage of vancomycin-resistant Enterococcus faecium: case series and review of literature. BMC Infectious Diseases, 14(1), 514. https://doi.org/10.1186/1471-2334-14-514

8. Cheng, V. C. C., Tai, J. W. M., Chau, P. H., Lai, C. K. C., Chuang, V. W. M., So, S. Y. C., ... Yuen, K. Y. (2016). Successful control of emerging vancomycin-resistant enterococci by territory-wide implementation of directly observed hand hygiene in patients in Hong Kong. American Journal of Infection Control, 44(10), 1168-1171. https://doi.org/10.1016/j.ajic.2016.03.050

9. Chiang, H.-Y., Perencevich, E. N., Nair, R., Nelson, R. E., Samore, M., Khader, K., ... Schweizer, M. L. (2017). Incidence and Outcomes Associated With Infections Caused by Vancomycin-Resistant Enterococci in the United States: Systematic Literature Review and Meta-Analysis. Infection Control \& Hospital Epidemiology, 38(02), 203-215. https://doi.org/10.1017/ice.2016.254

10. Contreras, G. A., Munita, J. M., \& Arias, C. A. (2019). Novel Strategies for the Management of Vancomycin-Resistant Enterococcal Infections. Current Infectious Disease Reports, 21(7). https://doi.org/10.1007/s11908-019-0680-y

11. Diario Oficial de la Federación. (2009). NORMA Oficial Mexicana NOM-045-SSA2-2005, Para la vigilancia epidemiológica, prevención y control de las infecciones nosocomiales. Al margen un sello con el Escudo Nacional, que dice: Estados Unidos Mexicanos.-Secretaría de Salud. Norma Oficial Mexicana Nom-045-Ssa2-2005, Para La Vigilancia Epidemiologica, Prevencion Y Control De Las Infecciones Nosocomiales. México, DF. Retrieved from http://www.ssm.gob.mx/portal/page/vig_epid/1/normas/NOM-045SSA2-2005, Para la vigilancia epidemiologica, prevencion y control de las infecciones nosocomiales.pdf

12. Djembi, L. F., Hodille, E., Chomat-Jaboulay, S., Coudrais, S., De Santis, N., Gardes, S., ... Girard, R. (2017). Factors associated with Vancomycin-resistant Enterococcus acquisition during a large outbreak. Journal of Infection and Public Health, 10, 185-190. https://doi.org/10.1016/j.jiph.2016.04.010

13. Domig, K. J., Mayer, H. K., \& Kneifel, W. (2003). Methods used for the isolation, enumeration, characterisation and identification of Enterococcus spp.: 1. Media for isolation and enumeration. International Journal of Food Microbiology, 88(2-3), 147-164. https://doi.org/10.1016/S0168-1605(03)00177-6 
14. Faron, M. L., Ledeboer, N. A., \& Buchan, B. W. (2016). Resistance Mechanisms, Epidemiology, and Approaches to Screening for Vancomycin-Resistant Enterococcus in the Health Care Setting. Journal of Clinical Microbiology, 54(10), 2436-2447. https://doi.org/10.1128/JCM.00211-16

15. Fernando, S. A., Gray, T. J., \& Gottlieb, T. (2017). Healthcareacquired infections: prevention strategies. Internal Medicine Journal, 47(12), 1341-1351. https://doi.org/10.1111/imj.13642

16. Flanagan, E., Cassone, M., Montoya, A., \& Mody, L. (2016). Infection Control in Alternative Health Care Settings: An Update. Infectious Disease Clinics of North America, 30(3), 785-804. https://doi.org/10.1016/j.idc.2016.05.001

17. Foolad, F., Taylor, B. D., Shelburne, S. A., Arias, C. A., \& Aitken, S. L. (2018). Association of daptomycin dosing regimen and mortality in patients with VRE bacteraemia: A review. Journal of Antimicrobial Chemotherapy, 73(9), 2277-2283. https://doi.org/10.1093/jac/dky072

18. Frakking, F. N. J., Bril, W. S., Sinnige, J. C., Klooster, J. E. va. t., de Jong, B. A. W., van Hannen, E. J., \& Tersmette, M. (2018). Recommendations for the successful control of a large outbreak of vancomycin-resistant Enterococcus faecium in a non-endemic hospital setting. Journal of Hospital Infection, S0195-6701, 30109-9. https://doi.org/10.1016/j.jhin.2018.02.016

19. Francisco, J., Aragão, I., \& Cardoso, T. (2018). Risk factors for longterm mortality in patients admitted with severe infection. BMC Infectious Diseases, 18(1), 161. https://doi.org/10.1186/s12879-0183054-4

20. Freitas, A. R., Tedim, A. P., Novais, C., Ruiz-Garbajosa, P., Werner, G., Laverde-Gomez, J. A., ... Coque, T. M. (2010). Global spread of the hylEfm colonization-virulence gene in megaplasmids of the Enterococcus faecium CC17 polyclonal subcluster. Antimicrobial Agents and Chemotherapy, 54(6), 2660-2665.

https://doi.org/10.1128/AAC.00134-10

21. Gelman, D., Beyth, S., Lerer, V., Adler, K., Poradosu-Cohen, R., Coppenhagen-Glazer, S., \& Hazan, R. (2018). Combined bacteriophages and antibiotics as an efficient therapy against VRE Enterococcus faecalis in a mouse model. Research in Microbiology, 169(9), 531-539. https://doi.org/10.1016/j.resmic.2018.04.008

22. Gilbert, E. M., Zembower, T. R., Rhodes, N. J., Qi, C., Reiner, S., Malczynski, M., \& Scheetz, M. H. (2017). Factors contributing to vancomycin-resistant Enterococcus spp. horizontal transmission events: exploration of the role of antibacterial consumption. 
Diagnostic Microbiology and Infectious Disease, 89(1), 72-77. https://doi.org/10.1016/J.DIAGMICROBIO.2017.05.014

23. Grabsch, E. A., Mahony, A. A., Cameron, D. R. M., Martin, R. D., Heland, M., Davey, P., ... Grayson, M. L. (2012). Significant reduction in vancomycin-resistant enterococcus colonization and bacteraemia after introduction of a bleach-based cleaning-disinfection programme. Journal of Hospital Infection, 82(4), 234-242. https://doi.org/10.1016/J.JHIN.2012.08.010

24. Hall, A. D., Steed, M. E., Arias, C. A., Murray, B. E., \& Rybak, M. J. (2012). Evaluation of standard- and high-dose daptomycin versus linezolid against vancomycin-resistant Enterococcus isolates in an in vitro pharmacokinetic/ pharmacodynamic model with simulated endocardial vegetations. Antimicrobial Agents and Chemotherapy, 56(6), 3174-3180. https://doi.org/10.1128/AAC.06439-11

25. Isenman, H., \& Fisher, D. (2016). Advances in prevention and treatment of vancomycin-resistant Enterococcus infection. Curr Opin Infect Dis, 29, 577-582.

https://doi.org/10.1097/QCO.0000000000000311

26. Kebriaei, R., Rice, S., Stamper, K., Dinh, A., Rios, R., Diaz, L., ... Ryback, M. (2018). crossm Influence of Inoculum Effect on the Efficacy of Daptomycin Monotherapy and in Combination with NL Lactams against. Antimicrobial Agents and Chemotherapy, 62(8), 112.

27. Kecik Bosnak, V., Namiduru, M., Karaoglan, I., \& Ozlem Mete, A. (2013). Evaluation of compliance in control and prevention study of vancomycin resistant Enterococcus outbreak. The Scientific World Journal, 2013, 252469. https://doi.org/10.1155/2013/252469

28. Kim, H. Y., Lee, W. K., Na, S., Roh, Y. H., Shin, C. S., \& Kim, J. (2016). The effects of chlorhexidine gluconate bathing on health careassociated infection in intensive care units: A meta-analysis. Journal of Critical Care, 32, 126-137.

https://doi.org/10.1016/J.JCRC.2015.11.011

29. Lai CK, Wong SY, Lee SS, Siu HK, Chiu CY, Tsang DN, Ip MP, H. C. (2017). A hospital-wide screening programme to control an outbreak of vancomycin-resistant enterococci in a large tertiary hospital in Hong Kong. Hong Kong Med J I, 23(2), 140-149. https://doi.org/10.12809/hkmj164939

30. Lee, T., Pang, S., Abraham, S., \& Coombs, G. W. (2019). Antimicrobial-resistant CC17 Enterococcus faecium: The past, the present and the future. Journal of Global Antimicrobial Resistance, 16, 36-47. https://doi.org/10.1016/j.jgar.2018.08.016 
31. Lemieux, C., Gardam, M., Evans, G., John, M., Suh, K. N., VanWalraven, C., ... Hota, S. (2017). Longitudinal Multicenter Analysis of Outcomes After Cessation of Control Measures for Vancomycin-Resistant Enterococci. Infection Control \& Hospital Epidemiology, 38(01), 24-30. https://doi.org/10.1017/ice.2016.235

32. Levitus, M., Rewane, A., \& Perera, T. B. (2021). VancomycinResistant Enterococci. StatPearls. StatPearls Publishing. Retrieved from http://www.ncbi.nlm.nih.gov/pubmed/30020605

33. Londoño Restrepo, J., Macias Ospina, I. C., \& Ochoa Jaramillo, F. L. (2016). Factores de riesgo asociados a infecciones por bacterias multirresistentes derivadas de la atención en salud en una institución hospitalaria de la ciudad de Medellín 2011-2014. Infectio, 20(2), 7783. https://doi.org/10.1016/j.infect.2015.09.002

34. Lowden, J., Miller Neilan, R., \& Yahdi, M. (2014). Optimal control of vancomycin-resistant enterococci using preventive care and treatment of infections. Mathematical Biosciences, 249, 8-17. https://doi.org/10.1016/J.MBS.2014.01.004

35. Mahony, A. A., Buultjens, A. H., Ballard, S. A., Grabsch, E. A., Xie, S., Seemann, T., ... Johnson, P. D. R. (2018). Vancomycin-resistant Enterococcus faecium sequence type 796 - rapid international dissemination of a new epidemic clone. Antimicrobial Resistance and Infection Control, 7(1). https://doi.org/10.1186/s13756-018-0335-z

36. Marra, A. R., Schweizer, M. L., \& Edmond, M. B. (2018). No-touch disinfection methods to decrease multidrug-resistant organism infections: A systematic review and meta-analysis. Infection Control and Hospital Epidemiology, 39(1), 20-31. https://doi.org/10.1017/ice.2017.226

37. Martin, E. M., Russell, D., Rubin, Z., Humphries, R., Grogan, T. R., Elashoff, D., \& Uslan, D. Z. (2016). Elimination of Routine Contact Precautions for Endemic Methicillin-Resistant Staphylococcus aureus and Vancomycin-Resistant Enterococcus: A Retrospective QuasiExperimental Study. Infection Control and Hospital Epidemiology, 37(11), 1323-1330. https://doi.org/10.1017/ice.2016.156

38. Mendes, E. T., Ranzani, O. T., Marchi, A. P., Silva, M. T. da, Filho, J. U. A., Alves, T., ... Costa, S. F. (2016). Chlorhexidine bathing for the prevention of colonization and infection with multidrug-resistant microorganisms in a hematopoietic stem cell transplantation unit over a 9-year period: Impact on chlorhexidine susceptibility. Medicine, 95(46), e5271. https://doi.org/10.1097/MD.0000000000005271

39. Miller, W. R., Munita, J. M., \& Arias, C. A. (2014). Mechanisms of antibiotic resistance in enterococci HHS Public Access. Expert Rev Anti Infect Ther, 12(10), 1221-1236. 
https://doi.org/10.1586/14787210.2014.956092

40. Miller, W. R., Murray, B. E., Rice, L. B., \& Arias, C. A. (2016, June). Vancomycin-Resistant Enterococci: Therapeutic Challenges in the 21st Century. Infectious Disease Clinics of North America. https://doi.org/10.1016/j.idc.2016.02.006

41. Montoya, A., Cassone, M., \& Mody, L. (2016). Infections in Nursing Homes Epidemiology and Prevention Programs. Clin Geriatr Med., 32, 585-607. https://doi.org/10.1016/j.cger.2016.02.004

42. Morgan DJ, Murthy R, Munoz-Price LS, Barnden M, Camins BC, Johnston BL, R., \& Z, Sullivan KV, Shane AL, Dellinger EP, Rupp ME, B. G. (2015). Reconsidering contact precautions for endemic methicillin-resistant Staphylococcus aureus and vancomycin-resistant Enterococcus. Infect Control Hosp Epidemiol, 36(11), 1163-1172. https://doi.org/10.1016/j.ajic.2015.06.003

43. Munita, J. M., Mishra, N. N., Alvarez, D., Tran, T. T., Diaz, L., Panesso, D., ... Arias, C. A. (2014). Failure of high-dose daptomycin for bacteremia caused by daptomycin-susceptible enterococcus faecium harboring liasr substitutions. Clinical Infectious Diseases, 59(9), 1277-1280. https://doi.org/10.1093/cid/ciu642

44. Mutters, N. T., Mersch-Sundermann, V., Mutters, R., Brandt, C., Schneider-Brachert, W., \& Frank, U. (2013). Control of the spread of vancomycin-resistant enterococci in hospitals: epidemiology and clinical relevance. Deutsches Arzteblatt International, 110(43), 725731. https://doi.org/10.3238/arztebl.2013.0725

45. O’Driscoll, T., \& Crank, C. W. (2015). Vancomycin-resistant enterococcal infections: epidemiology, clinical manifestations, and optimal management. Infection and Drug Resistance, 8, 217-230. https://doi.org/10.2147/IDR.S54125

46. Orsi, G. B., \& Ciorba, V. (2013). Vancomycin resistant enterococci healthcare associated infections. Ann Ig, 25(1), 485-492. https://doi.org/10.7416/ai.2013.1948

47. Ozback, H. A. (2018). A novel high-resolution melting analysis approach for rapid detection of vancomycin- resistant enterococci. Ann Saudi Med, 38, 200-207. https://doi.org/10.14260/jemds/2014/2083

48. Reyes, K., Bardossy, A. C., \& Zervos, M. (2016). VancomycinResistant Enterococci: Epidemiology, Infection Prevention, and Control. Infectious Disease Clinics of North America, 30(4), 953-965. https://doi.org/10.1016/j.idc.2016.07.009

49. Rubin, M. A., Samore, M. H., \& Harris, A. D. (2018). The Importance of Contact Precautions for Endemic Methicillin-Resistant 
Staphylococcus aureus and Vancomycin-Resistant Enterococci. JAMA, 319(9), 863-864. https://doi.org/10.1001/jama.2017.21122

50. Rutala, W. A., Weber, D. J., Healthcare, \& Infection Control Practices Advisory Committee. (2017). Guideline for Disinfection and Sterilization in Healthcare Facilities, 2008. Retrieved from https://www.cdc.gov/infectioncontrol/pdf/guidelines/disinfectionguidelines.pdf

51. Satilmis, L., Vanhems, P., \& Bénet, T. (2015). Outbreaks of Vancomycin-resistant enterococci in hospital settings: A systematic review and calculation of the basic reproductive number. Infection Control and Hospital Epidemiology, 37(3), 289-294. https://doi.org/10.1017/ice.2015.301

52. Shukla, B. S., Shelburne, S., Reyes, K., Kamboj, M., Lewis, J. D., Rincon, S. L., ... Arias, C. A. (2016). Influence of Minimum Inhibitory Concentration in Clinical Outcomes of Enterococcus faecium Bacteremia Treated with Daptomycin: Is it Time to Change the Breakpoint? Clinical Infectious Diseases, 62(12), 1514-1520. https://doi.org/10.1093/cid/ciw173

53. Talaga-Ćwiertnia, K., \& Bulanda, M. (2018). Analysis of the world epidemiological situation among vancomycin-resistant Enterococcus faecium infections and the current situation in Poland. Przeglad Epidemiologiczny, 72(1), 3-15. Retrieved from http://www.ncbi.nlm.nih.gov/pubmed/29664245

54. Tan, T. Y., Jiang, B., \& Ng, L. S. Y. (2017). Faster and economical screening for vancomycin-resistant enterococci by sequential use of chromogenic agar and real-time polymerase chain reaction. Journal of Microbiology, Immunology and Infection, 50(4), 448-453. https://doi.org/10.1016/j.jmii.2015.08.003

55. Ulrich, N., \& Gastmeier, P. (2017). Where is the difference between an epidemic and a high endemic level with respect to nosocomial infection control measures? An analysis based on the example of vancomycin-resistant Enterococcus faecium in hematology and oncology departments. GMS Hygiene and Infection Control, 12, 2196-5226. https://doi.org/10.3205/dgkh000299

56. Whang, D. W., Miller, L. G., Partain, N. M., \& McKinnell, J. A. (2013). Systematic review and meta-analysis of linezolid and daptomycin for treatment of vancomycin-resistant enterococcal bloodstream infections. Antimicrobial Agents and Chemotherapy, 57(10), 5013-5018. https://doi.org/10.1128/AAC.00714-13

57. Zhao, M., Liang, L., Ji, L., Chen, D., Zhang, Y., Zhu, Y., \& Patel, K. (2016). Similar efficacy and safety of daptomycin versus linezolid for treatment of vancomycin-resistant enterococcal bloodstream 
infections: a meta-analysis. International Journal of Antimicrobial Agents, 48(3), 231-238. https://doi.org/10.1016/J.IJANTIMICAG.2016.06.010

58. Ziakas, P. D., Thapa, R., Rice, L. B., \& Mylonakis, E. (2013). Trends and significance of VRE colonization in the ICU: a meta-analysis of published studies. PloS One, 8(9), e75658.

https://doi.org/10.1371/journal.pone.0075658 